\title{
La dyspepsie achlorhydrique
}

Les médecins on† été trop longtemps éduqués dans l'idée que, Гulcère gastro-duodénal étant associé à une hyperchlorhydrie, celle-ci est un symptôme de maladie d'estomac; aussi sont-ils rassurés par une absence de superacidité, et ne se préoccupent-ils pas de Thypo-chlorhydrie et de ses consequences.

Et pourtant, on considère aujourd'hui $\Gamma$ excès de secretion gastrique comme une reaction «normale» de la muqueuse irritée, done comme un signe de «bonne santé» de l'estomac! En revanche, Гanacidité * acquise correspond à une souffrance de la muqueuse, que ses efforts ont épuisée: toute gastrite chronique est done obligatoirement accom-pagnée d'hypochlorhydrie, et la conception de «gastritis super-acida» erronée.

Le fait qu'un grand nombre d'achyliques ne présente aucun trouble apparent - mais e'est le cas de nombreux hyperchlorhydriques aussi: les ulcéreux duodénaux, par exemple, entre deux poussées doulou-reuses! - a fait négliger ce groupe de dyspeptiques, et il faut être reconnaissant à B. F. Swynnerton et JV. C. Tanner d'etre revenus sur ce problème dans un gros article du British Medical Journal (6 mars 1954, p. 546 à 552).

Leur test d'anacidité est un repas d'épreuve au gruau, stimulant certes bien faible compare à Cliistamine; mais si cette dernière présente le grand avantage de permettre une épreuve fractionnée et de fournir un sue gastrique pur dont on peut facilement examiner le culot au microscope, une excitation aussi violente et artificielle procure une idee fausse des capacités sécrétoires de l'estomac: ce qui nous inté-resse, e'est de savoir comment l'estomac «répond» à rincitation physiologique du repas normal, et non à une drogue étrangère!

Sur 403 anacides observes au St. James's Hospital de Londres, les auteurs retiennent 2 groupes comprenant respectivement 113 et 126 malades, correspondant à ce qu'ils appellent «achlorhydríc patients with dyspepsía», c'est-à-dire dont les plaintes n'évoquent aucun * L' $\alpha$ privatif précédant une voyelle étant transformé en $\alpha v$ - pour des raisons euphoniques, «anacidité» est done correcternent compose. On en a, hélas, tire «<machlorhydrie» qui est une hérésie, puisque le préfixe $\alpha v \alpha$ - signifie «enhaut», «en arrière» ou «de nouveau», ou un non-sens si Ton admet an-achlorhydrie! Achlorhydrie est done seul correct, mais celà fait moins « scientifique $»$ !

134

Editorial

syndrome precis: malaises («discomfort») ou parfois douleurs épi-gastriques mises en relation avec les repas - y compris des brûlures interprétées comme «de Гacide» -; nausées et inappétence, renvois d'air, régurgitations, etc. Quand il s'y ajoute de Гanémie combinée à Гamaigrissement consécutif à $\Gamma$ inappétence, on pense évidemment au cancer. Quant aux troubles intestinaux, la diarrhée classique de l'achlorhydrie ne se rencontre pas plus fréquemment que la constipation: celle-ci prédominerait même largement dans les atrophies gastriques avancées. On n'est pas étonné d'apprendre que Гexamen gastroscopique révéla souvent la presence d'érosions sur cette muqueuse fragile; mais il y eut dans 11 cas des ulcères chroniques véritables, et des hémorragies manifestes chez un malade sur quatre! 
Les données exposées dans 12 tableaux de chiffres, les notions sur revolution vers le cancer ou Tanémie pernicieuse, les discussions pathogéniques, méritent une lecture attentive de cet article. II a laissé de côté cependant un problème encore plus frequent: la coexistence de troubles iléo-côliques chroniques et rebelles, sans manifestations épigastriques subjectives, et provenant d'une insuffisance sécrétoire digestive, dont $\Gamma$ achlorhydrie est la premiere étape, sinon le primum moyens. M. D.

Seite der Therapie - Page de thérapeutique Ravina, A.: Traitement de l'oxyurose par les antibiotiques. Presse Méd. 63, 728 (14 mai 1955).

Rien n'est plus décevant que de chercher à débarrasser un malade des oxyures qui infestent son intestin, les nombreux traitements proposes ces dernières années ne possédant qu'une efficacité très irrégulière.

Or on s'est aperçu que la terramycíne arrête le développement des vers, et les empêche de se reproduire; et dès 1951, Wells, puis Loughlín et leurs collaborateurs aux E.U.A. ont propose d'utiliser systématiquement ce medicament.

La posologie consiste en 7 jours de traitement, à raison de 1-2 g. par jour, selon Гâge (d'autres limitent la dose à $0,01-0,015 \mathrm{~g} . / \mathrm{kg}$.); et la cure ne nécessite aucune des mesures adjuvantes si astreignantes (lavements, ponimade périanale, etc.). Les æufs de parasites se modifient dès le deuxième jour, pour disparaître dès le sixième jour. Le traitement agirait en détruisant les femelles fécondées, en arrêtant le développement des æufs, et, de façon indirecte, en modifiant la flore intestinale.

La tétracycline semble avoir les mêmes qualités oxyuricides que la terramycine. Les travaux comparatifs de ces cernières années recommandant pourtant d'associer Tantibiotique à Vhydrate de pipérazíne (sirop à $10 \%, 1 / 2-2$ cuill. à cafe, trois fois par jour, selon l'âge) pendant deux semaines séparées par une semaine sans medicament. 